\title{
Simplex-Based Concrete Mix Design
}

\author{
C.E. Okere ${ }^{1}$, D.O. Onwuka ${ }^{2}$, S.U. Onwuka ${ }^{3}$, J.I. Arimanwa ${ }^{4}$ \\ ${ }^{1,2}$ Civil Engineering, Federal University of Technology, Owerri, Nigeria. \\ ${ }^{3}$ Project Management Technology, Federal University of Technology, Owerri, Nigeria. \\ ${ }^{4}$ Civil Engineering, Federal University of Technology, Owerri, Nigeria.
}

\begin{abstract}
Normal concrete is a mixture of cement, water, fine and coarse aggregates. Concrete mix design involves selecting the correct proportions of these constituent materials to produce concrete having the specified properties. Various mix design methods have some limitations. Time, energy and money are sometimes being wasted in order to get the appropriate mix proportions. In this paper, a mathematical model based on simplex method is formulated for the optimization of concrete cube strength. The model can provide all the possible mix ratios that can yield the desired concrete cube strength. It can also produce the concrete cube strength if mix proportions are given as well as the optimum value. Statistical tests were used to verify the adequacy of the model. They all agreed to the acceptance of the model.
\end{abstract}

Keywords: model; optimisation; concrete cube strength; simplex method.

\section{Introduction}

Concrete which is the most widely used construction material is composed of cement, water, fine and coarse aggregates.

Two main objectives of hardened concrete tests are control of quality and compliance with specifications [1]. Concrete cube strength test is one of the major tests carried out on concrete before it can be used effectively. Also concrete grades are usually specified in standard construction work.

Basically, the problem of designing a concrete mix consists of selecting the correct proportions of cement, fine and coarse aggregates and water to produce concrete having the specified properties [2]. Various methods have been developed in order to achieve the desired properties of concrete cube strength. These methods are time, money and energy consuming.

To minimize some of these limitations an optimization procedure has been proposed. It is a process that seeks for the maximum or minimum value of a function of several variables while at the same time, satisfying a number of other requirements [3]. In this paper, a mathematical model using simplex method is formulated for the optimisation of concrete cube strength.

\section{Methodology}

The main materials used in the work are cement, fine and coarse aggregates and water. the test.

Eagle cement, a brand of Ordinary Portland Cement, conforming to British Standard [4] was used in

The fine aggregate used in the work was river sand free from deleterious matters such as dirts, clay and organic matters. The fine aggregate falls into zone 3 of the grading curve.

The coarse aggregate was normal weight, irregular shaped coarse aggregate with a maximum size of $20 \mathrm{~mm}$. Both the fine and coarse aggregate were hard and durable, and conform to the specifications of British Standard [5].

Portable drinking water was used for the production of the concrete specimen tested.

Scheffe's simplex method was used in the optimisation of concrete mix design.

\section{Formulation of optimisation model based on Scheffe's simplex theory}

A simplex lattice is described as a structural representation of lines joining the atoms of a mixture .The atoms are constituent components of the mixture. For a normal concrete mixture, the constituent elements are water, cement, fine and coarse aggregates. And so it gives a simplex of a mixture of four components. Hence the simplex lattice of this four- component mixture is a three- dimensional solid equilateral tetrahedron. Mixture components are subject to the constraint that the sum of all the components must be equal to one [6].

In order words:

$$
\begin{gathered}
\mathrm{X}_{1}+\mathrm{X}_{2}+\mathrm{X}_{3}+\ldots \ldots \ldots+\mathrm{X}_{\mathrm{q}}=1 \\
\sum_{\mathrm{i}=1}^{\mathrm{q}} \mathrm{X}_{\mathrm{i}}=1
\end{gathered}
$$


Where $\mathrm{q}$ is the number of components of a mixture

$\mathrm{X}_{\mathrm{i}}$ is the proportion of the ith component in the mixture.

The $(\mathrm{q}, \mathrm{n})$ simplex lattice design introduced by Scheffe in 1958 [6], are characterized by the symmetric arrangements of points within the experimental region and a well chosen polynomial equation to represent the response surface over the entire simplex region. The response represents the property studied, namely, the concrete cube strength. The polynomial is obtained by using the restriction given by 'equation (1)' or 'equation (2)'.

A polynomial function of degree $\mathrm{n}$ in the $\mathrm{q}$ variables $\mathrm{X}_{1}, \mathrm{X}_{2}, \mathrm{X}_{3}, \ldots \ldots \ldots, \mathrm{X}_{\mathrm{q}}$ is given in form of

$$
\mathrm{y}=\mathrm{b}_{\mathrm{o}}+\sum \mathrm{b}_{\mathrm{i}} \mathrm{X}_{\mathrm{i}}+\sum \mathrm{b}_{\mathrm{ij}} \mathrm{X}_{\mathrm{i}} \mathrm{X}_{\mathrm{j}}+\sum \mathrm{b}_{\mathrm{ijk}} \mathrm{X}_{\mathrm{i}} \mathrm{X}_{\mathrm{j}} \mathrm{X}_{\mathrm{k}}+\sum \mathrm{bi}_{1} \mathrm{i}_{2} \ldots \ldots . \mathrm{i}_{\mathrm{n}} \mathrm{Xi}_{1} \mathrm{Xi}_{2} \ldots \ldots \mathrm{Xi}_{\mathrm{n}}
$$

where $\left(1 \leq \mathrm{i} \leq \mathrm{q}, 1 \leq \mathrm{i} \leq \mathrm{j} \leq \mathrm{q}, \quad 1 \leq \mathrm{i} \leq \mathrm{j} \leq \mathrm{k} \leq \mathrm{q}, \quad \mathrm{i} \leq \mathrm{i}_{1} \leq \mathrm{i}_{2} \leq \ldots \ldots \ldots . \mathrm{i}_{\mathrm{n}} \leq \mathrm{q}\right.$ respectively)

and $\mathrm{b}=$ constant coefficients

In general, the reduced form of 'equation (3)' is in the form of 'equation (4)' for a

polynomial function with $\mathrm{n}=4$.

$$
\begin{aligned}
Y= & b_{0}+b_{1} X_{1}+b_{2} X_{2}+b_{3} X_{3}+b_{4} X_{4}+b_{12} X_{1} X_{2}+b_{13} X_{1} X_{3} \\
& +b_{14} X_{1} X_{4}+b_{23} X_{2} X_{3}+b_{24} X_{2} X_{4}+b_{34} X_{3} X_{4}+b_{11} X_{1}^{2} \\
& +b_{22} X_{2}^{2}+b_{33} X_{3}^{2}+b_{44} X_{4}^{2}
\end{aligned}
$$

Multiplying 'equation (1)' by bo gives 'equation (5)'

$$
b_{0} X_{1}+b_{0} X_{2}+b_{0} X_{3}+b_{0} X_{4}=b_{0}
$$

Multiplying 'equation (1)' successively by $\mathrm{X}_{1}, \mathrm{X}_{2}, \mathrm{X}_{3}$, and $\mathrm{X}_{4}$ and rearranging gives 'equation (6)'

$$
\begin{aligned}
& X_{1}^{2}=X_{1}-X_{1} X_{2}-X_{1} X_{3}-X_{1} X_{4} \\
& X_{2}^{2}=X_{2}-X_{1} X_{2}-X_{2} X_{3}-X_{2} X_{4} \\
& X_{3}^{2}=X_{3}-X_{1} X_{3}-X_{2} X_{3}-X_{3} X_{4} \\
& X_{4}^{2}=X_{4}-X_{1} X_{4}-X_{2} X_{4}-X_{3} X_{4}
\end{aligned}
$$

Substituting 'equations (5) and (6)' into 'equation (4)' and simplifying yields 'equation (7)'

$$
\begin{gathered}
Y=\alpha_{1} X_{1}+\alpha_{2} X_{2}+\alpha_{3} X_{3}+\alpha_{4} X_{4}+\alpha_{12} X_{1} X_{2}+\alpha_{13} X_{1} X_{3}+\alpha_{14} X_{1} X_{4} \\
+\alpha_{23} X_{2} X_{3}+\alpha_{24} X_{2} X_{4}+\alpha_{34} X_{3} X_{4}
\end{gathered}
$$

Where the coefficients, $\alpha_{1}$ and $\alpha_{34}$ are defined in general as follows:

$$
\begin{gathered}
\alpha_{i}=b_{o}+b_{i}+b_{i i} \\
\alpha_{i j}=b_{i j}-b_{i i}-b_{j j}
\end{gathered}
$$

'Equation (7)' can be reduced further as follows:

$$
Y=\sum_{1 \leq i \leq q} \alpha_{i} X_{i}+\sum_{1 \leq i \leq j \leq q} \alpha_{i j} X_{i} X_{j}
$$

'Equation (9)' is the response to the pure component, $i$ and the binary mixture of components $i$ and $j$.

\section{Determination of the coefficients of the $(4,2)$ polynomial}

Assuming the response function for the pure component, $i$ and that for the binary mixture of components $i$ and $j$ are $y_{\mathrm{i}}$ and $\mathrm{y}_{\mathrm{ii}}$ respectively, then

$$
y_{i}=\sum_{i=1}^{4} \alpha_{i} X_{i}
$$

and

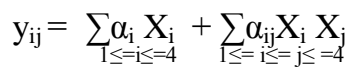

Substituting the values of $X_{1}, X_{2}, X_{3}$, and $X_{4}$ at the ith point (i.e. any of the vertices of the lattice) into 'equation (10)' gives the following general equation.

$$
y_{i}=\alpha_{i}
$$

For example, at point one, the value of $X_{1}=1$ while the values of $X_{2}, X_{3}$ and $X_{4}$ are equal to zero because $\sum X=$ 0 . Substituting the values of $\mathrm{X}_{1}, \mathrm{X}_{2}, \mathrm{X}_{3}$, and $\mathrm{X}_{4}$ into 'equation (10)' gives

$$
\mathrm{y}_{1}=\alpha_{1}
$$

Substituting the values of $\mathrm{X}_{1}, \mathrm{X}_{2}, \mathrm{X}_{3}$, and $\mathrm{X}_{4}$ at the point ij (that is at the mid point of the borderline connecting points $i$ and $j$ ) of the lattice, into 'equation (11)' yields:

$$
y_{i j}=1 / 2 \alpha_{i}+1 / 2 \alpha_{j}+1 / 4 \alpha_{i j}
$$

For point 12, that is at the midpoint of the borderlines connecting points 1 and 2 of the lattice, the values of $X_{1}=$ $X_{2}=1 / 2$ while the values of $X_{3}$, and $X_{4}$ are equal to zero because $\sum X_{i}=1$. Substituting the values of $X_{1}, X_{2}, X_{3}$, and $\mathrm{X}_{4}$ into 'equation (11)', gives 'equation (13b)'

From 'equation (12a)',

$$
\mathrm{y}_{12}=1 / 2 \alpha_{1}+1 / 2 \alpha_{2}+1 / 4 \alpha_{12}
$$


Similarly,

$$
\alpha_{\mathrm{i}}=\mathrm{y}_{\mathrm{i}}
$$

Rearranging 'equation (13a)' yields:

$$
\alpha_{j}=y_{j}
$$

Substituting 'equations (14) and (15)' into 'equation (16)' gives:

$$
\alpha_{\mathrm{ij}}=4 \mathrm{y}_{\mathrm{ij}}-2 \mathrm{y}_{\mathrm{i}}-2 \mathrm{y}_{\mathrm{j}}
$$

When 'equations (14), (15) and (16b)' are substituted, 'equation (7)' becomes:

$$
\begin{aligned}
\mathrm{y}= & \mathrm{y}_{1} \mathrm{X}_{1}+\mathrm{y}_{2} \mathrm{X}_{2}+\mathrm{y}_{3} \mathrm{X}_{3}+\mathrm{y}_{4} \mathrm{X}_{4}+\left(4 \mathrm{y}_{12}-2 \mathrm{y}_{1}-2 \mathrm{y}_{2}\right) \mathrm{X}_{1} \mathrm{X}_{2} \\
& +\left(4 \mathrm{y}_{13}-2 \mathrm{y}_{1}-2 \mathrm{y}_{3}\right) \mathrm{X}_{2} \mathrm{X}_{3}+\left(4 \mathrm{y}_{14}-2 \mathrm{y}_{1}-2 \mathrm{y}_{4}\right) \mathrm{X}_{1} \mathrm{X}_{4} \\
& +\left(4 \mathrm{y}_{23}-2 \mathrm{y}_{2}-2 \mathrm{y}_{3}\right) \mathrm{X}_{2} \mathrm{X}_{3}+\left(4 \mathrm{y}_{24}-2 \mathrm{y}_{2}-2 \mathrm{y}_{4}\right) \mathrm{X}_{2} \mathrm{X}_{4} \\
& +\left(4 \mathrm{y}_{34}-2 \mathrm{y}_{3}-2 \mathrm{y}_{4}\right) \mathrm{X}_{3} \mathrm{X}_{4}
\end{aligned}
$$

Let the coefficient of $y_{1}=X_{1}--2 X_{1}\left(X_{2}+X_{3}+X_{4}\right)$

From 'equation (1)',

$$
\mathrm{X}_{2}+\mathrm{X}_{3}+\mathrm{X}_{4}=1-\mathrm{X}_{1}
$$

Substituting 'equation (19)' into equation (18)' gives the coefficient of $y_{1}$ as follows:

$$
\begin{aligned}
\mathrm{y}_{1} & =\mathrm{X}_{1}-2 \mathrm{X}_{1}\left(1-\mathrm{X}_{1}\right) \\
& =\mathrm{X}_{1}\left(2 \mathrm{X}_{1}-1\right)
\end{aligned}
$$

Rearranging 'equation (17)' and transferring all the coefficients of $\mathrm{y}_{1}$ in like manner, gives the following mixture design model for optimization of a 4-component concrete.

$$
\begin{gathered}
\mathrm{y}=\mathrm{X}_{1}\left(2 \mathrm{X}_{1}-1\right) \mathrm{y}_{1}+\mathrm{X}_{2}\left(2 \mathrm{X}_{2}-1\right) \mathrm{y}_{2}+\mathrm{X}_{3}\left(2 \mathrm{X}_{3}-1\right) \mathrm{y}_{3} \\
+\mathrm{X}_{4}\left(2 \mathrm{X}_{4}-1\right) \mathrm{y}_{4}+4 \mathrm{X}_{1} \mathrm{X}_{2} \mathrm{y}_{12}+4 \mathrm{X}_{1} \mathrm{X}_{3} \mathrm{y}_{13}+4 \mathrm{X}_{1} \mathrm{X}_{4} \mathrm{y}_{14} \\
+4 \mathrm{X}_{2} \mathrm{X}_{3} \mathrm{y}_{23}+4 \mathrm{X}_{2} \mathrm{X}_{4} \mathrm{y}_{24}+4 \mathrm{X}_{3} \mathrm{X}_{4} \mathrm{y}_{14}
\end{gathered}
$$

The terms $y_{i}$ and $y_{i i}$ are responses (representing concrete cube strength) at the points $i$ and ij. They are determined by carrying out laboratory test.

\section{Components transformation}

It is impossible to use the normal mix ratios such as 1:2:4 or 1:3:6 at given water/cement ratio because of the requirement of the simplex that sum of all the components must be one. Hence it is necessary to carry out a transformation from actual to pseudo components. The actual components represent the proportion of the ingredients while the pseudo components represent the proportion of the components of the ith component in the mixture i.e. $\mathrm{X}_{1}, \mathrm{X}_{2}, \mathrm{X}_{3}, \mathrm{X}_{4}$. Considering the four-component mixture tetrahedron simplex lattice, let the vertices of this tetrahedron (principal coordinates) be described by $A_{1}, A_{2}, A_{3}, A_{4}$.

The arbitrary mix proportions prescribed for the vertices of the tetrahedron shown in "Figure 1",

$\mathrm{A}_{1}(0.55: 1: 2: 4)$

$\mathrm{A}_{2}(0.50: 1: 2.5: 6)$

$\mathrm{A}_{3}(0.45: 1: 3: 5.5)$

$\mathrm{A}_{4}(0.6: 1: 1.5: 3.5)$

are based on past experiences and literature.

$\mathrm{A}_{1}(0.55,1,2,4)$

$\mathrm{A}_{2}(0.5,1,2.5,6)$

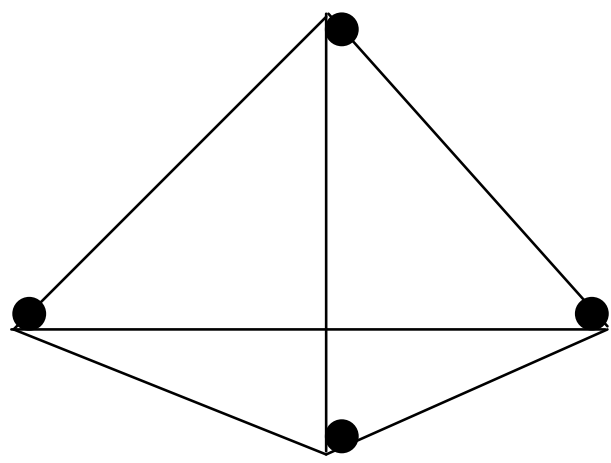

$\mathrm{A}_{4}(0.6,1,1.5,3.5)$

$\mathrm{A}_{3}(0.45,1,3,5.5)$

Fig 1: Vertices of a $(4,2)$ lattice (actual) 


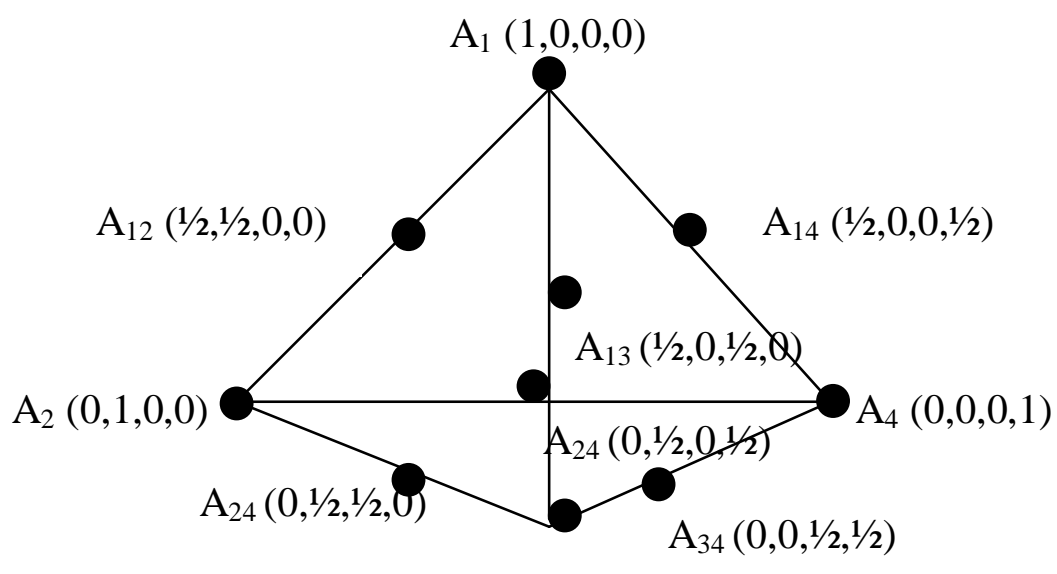

$\mathrm{A}_{3}(0.0,0,1,0)$

Fig 2: Vertices of a $(4,2)$ lattice (pseudo)

Let $\mathrm{X}$ represent pseudo components and $\mathrm{Z}$, actual components. For component transformation we use the following equations:

$$
\begin{aligned}
& \mathrm{X}=\mathrm{BZ} \\
& \mathrm{Z}=\mathrm{AX}
\end{aligned}
$$

where $\mathrm{A}=$ matrix whose elements are from the arbitrary mix proportions chosen when 'equation (23)' is opened and solved mathematically.

$\mathrm{B}=$ the inverse of matrix A

$\mathrm{Z}=$ matrix of actual components

$\mathrm{X}=$ matrix of pseudo components obtained from "Figure 2".

Expanding and using 'equations (22) and (23)' the actual components $Z$ were determined and presented in Table 1.

Table 1. Actual Components Z

\begin{tabular}{|l|l|l|l|l|l|l|l|l|l|}
\hline $\mathrm{N}$ & $\mathrm{X}_{1}$ & $\mathrm{X}_{2}$ & $\mathrm{X}_{3}$ & $\mathrm{X}_{4}$ & Response & $\mathrm{Z}_{1}$ & $\mathrm{Z}_{2}$ & $\mathrm{Z}_{3}$ & $\mathrm{Z}_{4}$ \\
\hline 1 & 1 & 0 & 0 & 0 & $\mathrm{Y}_{1}$ & 0.55 & 1 & 2 & 4 \\
2 & 0 & 1 & 0 & 0 & $\mathrm{Y}_{2}$ & 0.50 & 1 & 2.5 & 6 \\
3 & 0 & 0 & 1 & 0 & $\mathrm{Y}_{3}$ & 0.45 & 1 & 3 & 5.5 \\
4 & 0 & 0 & 0 & 1 & $\mathrm{Y}_{4}$ & 0.6 & 1 & 1.5 & 3.5 \\
5 & 0.5 & 0.5 & 0 & 0 & $\mathrm{Y}_{12}$ & 0.525 & 1 & 2.25 & 5 \\
6 & 0.5 & 0 & 0.5 & 0 & $\mathrm{Y}_{13}$ & 0.5 & 1 & 2.5 & 4.75 \\
7 & 0.5 & 0 & 0 & 0.5 & $\mathrm{Y}_{14}$ & 0.575 & 1 & 1.75 & 3.75 \\
8 & 0 & 0.5 & 0.5 & 0 & $\mathrm{Y}_{23}$ & 0.475 & 1 & 2.75 & 5.75 \\
9 & 0 & 0.5 & 0 & 0.5 & $\mathrm{Y}_{24}$ & 0.55 & 1 & 2 & 4.75 \\
10 & 0 & 0 & 0.5 & 0.5 & $\mathrm{Y}_{34}$ & 0.525 & 1 & 2.25 & 4.5 \\
\hline
\end{tabular}

Control points
\begin{tabular}{|l|l|l|l|l|l|l|l|l|l|}
\hline 11 & 0.5 & 0.25 & 0.25 & 0 & $\mathrm{C}_{1}$ & 0.5125 & 1 & 2.375 & 4.875 \\
12 & 0.25 & 0.25 & 0.25 & 0.25 & $\mathrm{C}_{2}$ & 0.525 & 1 & 2.25 & 4.75 \\
13 & 0 & 0.25 & 0.25 & 0.5 & $\mathrm{C}_{3}$ & 0.5375 & 1 & 2.125 & 4.625 \\
14 & 0 & 0.25 & 0 & 0.75 & $\mathrm{C}_{4}$ & 0.575 & 1 & 1.75 & 4.125 \\
15 & 0.75 & 0 & 0.25 & 0 & $\mathrm{C}_{5}$ & 0.525 & 1 & 2.25 & 4.375 \\
16 & 0 & 0.5 & 0.25 & 0.25 & $\mathrm{C}_{6}$ & 0.5125 & 1 & 2.375 & 5.25 \\
17 & 0.25 & 0 & 0.5 & 0.25 & $\mathrm{C}_{7}$ & 0.5125 & 1 & 2.375 & 4.625 \\
18 & 0.75 & 0.25 & 0 & 0 & $\mathrm{C}_{8}$ & 0.5375 & 1 & 2.125 & 4.5 \\
19 & 0 & 0.75 & 0.25 & 0 & $\mathrm{C}_{9}$ & 0.4875 & 1 & 2.625 & 5.875 \\
20 & 0 & 0.4 & 0.4 & 0.2 & $\mathrm{C}_{10}$ & 0.5 & 1 & 2.5 & 5.3 \\
\hline
\end{tabular}

If there is need to use bulk volume in the mix design, one has to carry out components transformation as stated above using equations '(22) and (23)'. It is worthy of note here that the equation derived has no need of the value of the specific gravity. 


\section{Experimental method}

The actual components as transformed from 'equation (5)' and (Table 1) were used to measure out the quantities water $\left(Z_{1}\right)$, cement $\left(Z_{2}\right)$, sand $\left(Z_{3}\right)$, and coarse aggregates $\left(Z_{4}\right)$ in their respective ratios for the concrete cube strength test. For instance, the actual ratio for the test number 20 means that the concrete mix ratio is 1: $2.5: 5.3$ at 0.5 free water/cement ratio. A total of $20 \mathrm{mix}$ ratios were used to produce 40 prototype concrete cubes measuring $150 \mathrm{~mm} \times 150 \mathrm{~mm} \times 150 \mathrm{~mm}$ that were cured and tested on the $28^{\text {th }}$ day. Ten out of $20 \mathrm{mix}$ ratios were used as control mix ratios to produce 20 cubes for the confirmation of the adequacy of the mixture design model given by 'equation (21)'. The cubes were then tested for concrete cube strength using the universal testing machine. The load under which the cube specimen failed was recorded and used to compute the strength of the concrete cubes.

\section{Results and analysis}

Table 2).

The test result of the concrete cube strength $\left(\mathrm{Y}_{\mathrm{i}}\right)$ based on day 28-day strength, is presented as part of

The concrete cube strength was obtained from the following equation:

$$
f_{c u}=P / A
$$

where $\mathrm{f}_{\mathrm{cu}}$ is the concrete cube strength in Mega Pascals (MPa) or Newtons per millimeters squared $\left(\mathrm{Nmm}^{-2}\right)$. $\mathrm{P}=$ failure load in Newtons $(\mathrm{N})$.

$\mathrm{A}=$ nominal cross-sectional area in millimetres squared $\left(\mathrm{Nmm}^{-2}\right)$.

Table 2. Test Results and Replication Variance

\begin{tabular}{|c|c|c|c|c|c|c|c|}
\hline $\begin{array}{l}\text { Exp } \\
\text { No. }\end{array}$ & Replicates & $\begin{array}{ll}\begin{array}{l}\text { Response } \\
\left(\mathrm{N} / \mathrm{mm}^{2}\right)\end{array} & \mathrm{Y}_{\mathrm{i}} \\
\end{array}$ & $\begin{array}{l}\text { Response } \\
\text { Symbol }\end{array}$ & $\mathrm{Y}$ & $\sum \mathrm{Y}_{\mathrm{i}}$ & $\sum Y_{i}^{2}$ & $\mathrm{~S}_{\mathrm{i}}^{2}$ \\
\hline 1 & $\begin{array}{l}1 \mathrm{~A} \\
\mathrm{IB}\end{array}$ & $\begin{array}{l}27.10 \\
25.34\end{array}$ & $\mathrm{Y}_{1}$ & 26.22 & 54.44 & 1376.53 & 1.55 \\
\hline 2 & $\begin{array}{l}2 \mathrm{~B} \\
2 \mathrm{~B}\end{array}$ & $\begin{array}{c}31.12 \\
29.32 \\
\end{array}$ & $\mathrm{Y}_{2}$ & 30.22 & 60.44 & 1828.12 & 1.62 \\
\hline 3 & $\begin{array}{l}3 \mathrm{~A} \\
3 \mathrm{~B}\end{array}$ & $\begin{array}{l}25.20 \\
22.80 \\
\end{array}$ & $\mathrm{Y}_{3}$ & 24 & 48.00 & 1154.88 & 2.88 \\
\hline 4 & $\begin{array}{l}4 \mathrm{~A} \\
4 \mathrm{~B}\end{array}$ & $\begin{array}{l}27.90 \\
27.20\end{array}$ & $\mathrm{Y}_{4}$ & 27.55 & 55.10 & 1518.25 & 0.25 \\
\hline 5 & $\begin{array}{l}5 \mathrm{~A} \\
5 \mathrm{~B}\end{array}$ & $\begin{array}{l}27.58 \\
30.22\end{array}$ & $\mathrm{Y}_{12}$ & 28.89 & 57.78 & 1672.70 & 3.44 \\
\hline 6 & $\begin{array}{l}6 \mathrm{~A} \\
6 \mathrm{~B}\end{array}$ & $\begin{array}{l}23.31 \\
25.57\end{array}$ & $\mathrm{Y}_{13}$ & 24.44 & 48.88 & 1197.18 & 2.55 \\
\hline 7 & $\begin{array}{l}7 \mathrm{~A} \\
7 \mathrm{~B}\end{array}$ & $\begin{array}{l}20.13 \\
23.41\end{array}$ & $\mathrm{Y}_{14}$ & 21.77 & 43.54 & 953.25 & 5.38 \\
\hline 8 & $\begin{array}{l}8 \mathrm{~A} \\
8 \mathrm{~B}\end{array}$ & $\begin{array}{l}33.01 \\
29.21\end{array}$ & $\mathrm{Y}_{23}$ & 31.11 & 62.22 & 1942.88 & 7.22 \\
\hline 9 & $\begin{array}{l}9 \mathrm{~A} \\
9 \mathrm{~B}\end{array}$ & $\begin{array}{l}23.22 \\
21.66\end{array}$ & $\mathrm{Y}_{24}$ & 22.44 & 44.88 & 1008.32 & 1.21 \\
\hline 10 & $\begin{array}{l}10 \mathrm{~A} \\
10 \mathrm{~B}\end{array}$ & $\begin{array}{l}26.88 \\
25.12\end{array}$ & $\mathrm{Y}_{34}$ & 26.00 & 52.00 & 1008.32 & 1.55 \\
\hline 11 & $\begin{array}{l}11 \mathrm{~A} \\
11 \mathrm{~B}\end{array}$ & $\begin{array}{l}22.22 \\
29.40\end{array}$ & $\mathrm{C}_{1}$ & 25.81 & 51.62 & 1358.09 & 25.77 \\
\hline 12 & $\begin{array}{l}12 \mathrm{~A} \\
12 \mathrm{~B}\end{array}$ & $\begin{array}{l}22.22 \\
30.56 \\
\end{array}$ & $\mathrm{C}_{2}$ & 26.39 & 52.78 & 1427.64 & 34.78 \\
\hline 13 & $\begin{array}{l}13 \mathrm{~A} \\
13 \mathrm{~B}\end{array}$ & $\begin{array}{l}26.67 \\
27.29\end{array}$ & $\mathrm{C}_{3}$ & 26.98 & 53.96 & 1456.03 & 0.19 \\
\hline 14 & $\begin{array}{l}14 \mathrm{~A} \\
14 \mathrm{~B}\end{array}$ & $\begin{array}{l}23.78 \\
22.86 \\
\end{array}$ & $\mathrm{C}_{4}$ & 23.32 & 46.64 & 1088.07 & 0.43 \\
\hline 15 & $\begin{array}{l}15 \mathrm{~A} \\
15 \mathrm{~B}\end{array}$ & $\begin{array}{l}28.01 \\
28.47\end{array}$ & $\mathrm{C}_{5}$ & 28.24 & 56.48 & 1595.10 & 0.10 \\
\hline 16 & $\begin{array}{l}16 \mathrm{~A} \\
16 \mathrm{~B}\end{array}$ & $\begin{array}{l}29.33 \\
26.17\end{array}$ & $\mathrm{C}_{6}$ & 27.75 & 55.50 & 1545.12 & 4.99 \\
\hline 17 & $\begin{array}{l}17 \mathrm{~A} \\
17 \mathrm{~B}\end{array}$ & $\begin{array}{c}20.00 \\
27.38\end{array}$ & $\mathrm{C}_{7}$ & 23.69 & 47.38 & 1149.66 & 27.23 \\
\hline
\end{tabular}




\begin{tabular}{|l|l|l|l|l|l|l|l|}
\hline 18 & $18 \mathrm{~A}$ & 26.44 & $\mathrm{C}_{8}$ & 24.02 & 48.04 & 1165.63 & 11.71 \\
& $18 \mathrm{~B}$ & 21.60 & & & & & \\
\hline 19 & $19 \mathrm{~A}$ & 22.22 & $\mathrm{C}_{9}$ & 25.96 & 51.92 & 1375.82 & 27.98 \\
& $19 \mathrm{~B}$ & 29.70 & & & & & \\
\hline 20 & $20 \mathrm{~A}$ & 24.66 & $\mathrm{C}_{10}$ & 26.57 & 53.14 & 1419.23 & 7.30 \\
& $20 \mathrm{~B}$ & 28.48 & & & $\sum$ & 168.13 \\
\hline
\end{tabular}

The values of the mean of responses, $\mathrm{Y}$ and the variances of replicates $\mathrm{S}_{\mathrm{i}}{ }^{2}$ presented in columns 5 and 8 of (Table 2) were gotten from the following 'equations (25) and (26)':

$$
\begin{gathered}
\mathrm{Y}=\sum_{\mathrm{i}=1}^{\mathrm{n}} \mathrm{Y}_{\mathrm{i}} / \mathrm{n} \\
\mathrm{S}_{\mathrm{i}}^{2}=[1 /(\mathrm{n}-1)]\left\{\sum \mathrm{Y}_{\mathrm{i}}^{2}-\left[1 / \mathrm{n}\left(\sum \mathrm{Y}_{\mathrm{i}}\right)^{2}\right]\right\}
\end{gathered}
$$

Where $1 \leq \mathrm{i} \leq \mathrm{n}$ and this equation is an expanded form of 'equation(27)'

$$
\mathrm{S}_{\mathrm{i}}^{2}=[1 /(\mathrm{n}-1)]\left[\sum_{\mathrm{i}=1}^{\mathrm{n}}\left(\mathrm{Y}_{\mathrm{i}}-\mathrm{Y}\right)^{2}\right]
$$

Where $Y_{i}=$ responses

$\mathrm{Y}=$ mean of the responses for each control point

$\mathrm{n}=$ number of parallel observations at every point

$\mathrm{n}-1=$ degree of freedom

$\mathrm{S}_{\mathrm{i}}^{2}=$ variance at each design point

Considering all the design points, number of degrees of freedom,

$$
\begin{aligned}
\mathrm{V}_{\mathrm{e}} & =\sum \mathrm{N}-1 \\
& =20-1 \\
& =19
\end{aligned}
$$

Where $\mathrm{N}$ is the number of points

Replication variance, $S_{y}^{2}=(1 / \mathrm{Ve}) \sum_{\mathrm{i}=1}^{\mathrm{N}} \mathrm{S}_{\mathrm{i}}^{2}$

Where $\mathrm{S}_{\mathrm{i}}^{2}$ is the variance at each point

$$
=168.13 / 19=8.848
$$

Using 'equations (28) and (29)', the replication error, $S_{y}$ can be determined as follows

$$
\begin{aligned}
\mathrm{S}_{\mathrm{y}} & =\sqrt{ } \mathrm{S}_{\mathrm{y}}^{2} \\
& =2.97
\end{aligned}
$$

This replication error value was used below to determine the t-statistics values for Scheffe's simplex model.

Determination of the optimisation model based on Scheffe's theory as follows:

Using 'equation (31)' and (Table 2), the coefficients of the second degree polynomial were determined

$$
\alpha_{1}=y_{1} \text { and } \alpha_{i j}=4 y_{i j}-2 y_{i}-2 y_{j}
$$

$$
\begin{aligned}
& \alpha_{1}=26.22, \alpha_{2}=30.22, \alpha_{3}=24, \text { and } \alpha_{4}=27.55, \\
& \alpha_{12}=4(28.89)-2(26.22)-2(30.22)=2.68 \\
& \alpha_{13}=4(24.44)-2(26.22)-2(24)=-2.68 \\
& \alpha_{14}=4(21.77)-2(26.22)-2(27.55)=-20.46 \\
& \alpha_{23}=4(31.11)-2(30.22)-2(24)=16 \\
& \alpha_{24}=4(22.44)-2(30.22)-2(27.55)=25.78 \\
& \alpha_{34}=4(26)-2(24)-2(27.55)=0.9
\end{aligned}
$$

Substituting the values of these coefficients into 'equation (21)' yields:

$$
\begin{aligned}
\mathrm{Y}= & 26.22 \mathrm{X}_{1}+30.22 \mathrm{X}_{2}+24 \mathrm{X}_{3}+27.55 \mathrm{X}_{4}+2.68 \mathrm{X}_{1} \mathrm{X}_{2}-2.68 \mathrm{X}_{1} \mathrm{X}_{3}-20.46 \mathrm{X}_{1} \mathrm{X}_{4} \\
& +16 \mathrm{X}_{2} \mathrm{X}_{3}-25.78 \mathrm{X}_{2} \mathrm{X}_{4}+0.9 \mathrm{X}_{3} \mathrm{X}_{4}
\end{aligned}
$$

'Equation (32)' is the Scheffe's mathematical model for concrete cube strength based on the 28-day strength. 


\section{Test of the adequacy of the model}

The model equation was tested for adequacy against the controlled experimental results. The statistical hypothesis for this mathematical model is as follows:

Null Hypothesis $\left(\mathrm{H}_{0}\right)$ : There is no significant difference between the experimental and the theoretically expected results at an $\alpha$-level of 0.5 .

Alternative Hypothesis $\left(\mathrm{H}_{1}\right)$ : There is a significant difference between

the experimental and theoretically expected results at an $\alpha$-level of 0.05 .

The student's t-test and fisher test statistics were used for this test. The expected values ( $\mathrm{Y}_{\text {predicted }}$ ) for the test control points were obtained by substituting the values of $\mathrm{X}_{1}$ from (Table 1) into the model equation ie 'equation (32)'. These values were compared with the experimental result ( $\left.\mathrm{Y}_{\text {observed }}\right)$ given in (Table 2).

\section{Student's t-test}

For this test, the parameters $\Delta_{\mathrm{y}}, \mathrm{C}$ and $\mathrm{t}$ are evaluated using the following equations respectively

$$
\begin{aligned}
& \Delta_{\mathrm{Y}}=\mathrm{Y}_{\text {(observed) }}-\mathrm{Y}_{\text {(predicted) }} \\
& \mathrm{C}=\left(\sum \mathrm{a}_{\mathrm{i}}^{2}+\sum \mathrm{a}_{\mathrm{ij}}{ }^{2}\right) \\
& \mathrm{t}=\Delta_{\mathrm{y}} V_{\mathrm{n}} /[\mathrm{Sy} \sqrt{ }(1+\mathrm{C})]
\end{aligned}
$$

where $C$ is the estimated standard deviation or error,

$\mathrm{t}$ is the $\mathrm{t}$-statistics,

$\mathrm{n}$ is the number of parallel observations at every point

$\mathrm{S}_{\mathrm{y}}$ is the replication error

$\mathrm{a}_{\mathrm{i}}$ and $\mathrm{a}_{\mathrm{ij}}$ are coefficients while $\mathrm{i}$ and $\mathrm{j}$ are pure components

$\mathrm{a}_{\mathrm{i}}=\mathrm{X}_{\mathrm{i}}\left(2 \mathrm{X}_{\mathrm{i}}-1\right)$

$\mathrm{a}_{\mathrm{ij}}=4 \mathrm{X}_{\mathrm{i}} \mathrm{X}_{\mathrm{j}}$

$\mathrm{Y}_{\mathrm{obs}}=\mathrm{Y}_{\text {(observed })}=$ Experimental results

\begin{tabular}{|c|c|c|c|c|c|c|c|c|c|c|c|c|}
\hline $\mathrm{N}$ & $\mathrm{CN}$ & $\mathrm{i}$ & $\mathrm{j}$ & $\mathrm{a}_{\mathrm{i}}$ & $a_{i j}$ & $a_{i}^{2}$ & $a^{2}{ }_{i j}$ & $\epsilon$ & $\mathrm{Y}_{\mathrm{obs}}$ & $Y_{\text {pre }}$ & $\underline{\Delta}_{\mathrm{Y}}$ & $\mathrm{t}$ \\
\hline \multirow{8}{*}{1} & \multirow{8}{*}{$\mathrm{C}_{1}$} & 1 & 2 & 0 & 0.5 & 0 & 0.25 & \multirow[b]{8}{*}{0.6093} & \multirow{8}{*}{25.81} & \multirow{8}{*}{26.21} & \multirow{8}{*}{-0.4} & \multirow{8}{*}{-0.15} \\
\hline & & 1 & 3 & 0 & 0.5 & 0 & 0.25 & & & & & \\
\hline & & 1 & 4 & 0 & 0 & 0 & 0 & & & & & \\
\hline & & 2 & 3 & -0.125 & 0.25 & 0.0156 & 0.0625 & & & & & \\
\hline & & 2 & 4 & -0.125 & 0 & 0.0156 & 0 & & & & & \\
\hline & & 3 & 4 & -0.125 & 0 & 0.0156 & 0 & & & & & \\
\hline & & 4 & - & 0 & - & 0 & 0 & & & & & \\
\hline & & & & & $\sum$ & 0.0468 & 0.5625 & & & & & \\
\hline \multirow{8}{*}{2} & \multirow{7}{*}{$\mathrm{C}_{2}$} & 1 & 2 & -0.125 & 0.25 & 0.0156 & 0.0625 & \multirow[b]{8}{*}{0.4842} & \multirow{8}{*}{26.39} & \multirow{8}{*}{25.85} & \multirow{8}{*}{0.54} & \multirow{8}{*}{0.21} \\
\hline & & 1 & 3 & -0.125 & 0.25 & 0.0156 & 0.0625 & & & & & \\
\hline & & 1 & 4 & -0.125 & 0.25 & 0.0156 & 0.0625 & & & & & \\
\hline & & 2 & 3 & -0.125 & 0.25 & 0.0156 & 0.0625 & & & & & \\
\hline & & 2 & 4 & -0.125 & 0.25 & 0.0156 & 0.0625 & & & & & \\
\hline & & 3 & 4 & -0.125 & 0.25 & 0.0156 & 0.0625 & & & & & \\
\hline & & 4 & - & -0.125 & - & 0.0156 & - & & & & & \\
\hline & & & & & $\sum$ & 0.1092 & 0.375 & & & & & \\
\hline \multirow{8}{*}{3} & \multirow{8}{*}{$\mathrm{C}_{3}$} & 1 & 2 & 0 & 0 & 0 & 0 & \multirow[b]{8}{*}{0.6093} & \multirow{8}{*}{26.98} & \multirow{8}{*}{25.22} & \multirow{8}{*}{1.76} & \multirow{8}{*}{0.66} \\
\hline & & 1 & 3 & 0 & 0 & 0 & 0 & & & & & \\
\hline & & 1 & 4 & 0 & 0 & 0 & 0 & & & & & \\
\hline & & 2 & 3 & -0.125 & 0.25 & 0.0156 & 0.0625 & & & & & \\
\hline & & 2 & 4 & -0.125 & 0.5 & 0.0156 & 0.25 & & & & & \\
\hline & & 3 & 4 & -0.125 & 0.5 & 0.0156 & 0.25 & & & & & \\
\hline & & 4 & - & 0 & - & 0 & - & & & & & \\
\hline & & & & & $\sum$ & 0.0468 & 0.5625 & & & & & \\
\hline \multirow{6}{*}{4} & \multirow{6}{*}{$\mathrm{C}_{4}$} & 1 & 2 & 0 & 0 & 0 & 0 & & \multirow{6}{*}{23.32} & \multirow{6}{*}{23.38} & \multirow{6}{*}{-0.06} & \multirow{6}{*}{-0.02} \\
\hline & & 1 & 3 & 0 & 0 & 0 & 0 & & & & & \\
\hline & & 1 & 4 & 0 & 0 & 0 & 0 & & & & & \\
\hline & & 2 & 3 & -0.125 & 0 & 0.0156 & 0 & & & & & \\
\hline & & 2 & 4 & -0.125 & 0.75 & 0.0156 & 0.5625 & & & & & \\
\hline & & 3 & 4 & 0 & 0 & 0 & 0 & & & & & \\
\hline
\end{tabular}

$\mathrm{Y}_{\mathrm{pre}}=\mathrm{Y}_{(\text {predicted })}=$ Predicted results

Table 3. T-Statistics for test control points 


\begin{tabular}{|c|c|c|c|c|c|c|c|c|c|c|c|c|}
\hline & & 4 & - & 0.375 & - & 0.1406 & - & \multirow[b]{2}{*}{0.7343} & & & & \\
\hline & & & & & $\sum$ & 0.1718 & 0.5625 & & & & & \multirow{9}{*}{0.75} \\
\hline \multirow{8}{*}{5} & \multirow{8}{*}{$\mathrm{C}_{5}$} & 1 & 2 & 0.375 & 0 & 0.1406 & 0 & & \multirow{8}{*}{28.24} & \multirow{8}{*}{26.01} & \multirow{8}{*}{2.23} & \\
\hline & & 1 & 3 & 0.375 & 0.75 & 0.1406 & 0.5625 & & & & & \\
\hline & & 1 & 4 & 0.375 & 0 & 0.1406 & & & & & & \\
\hline & & 2 & 3 & 0 & 0 & 0 & 0 & & & & & \\
\hline & & 2 & 4 & & 0 & 0 & 0 & & & & & \\
\hline & & 3 & 4 & -0.125 & 0 & 0.0156 & 0 & & & & & \\
\hline & & & - & & - & & & & & & & \\
\hline & & & & & $\sum$ & 0.4374 & 0.5625 & 0.9999 & & & & \\
\hline \multirow{8}{*}{6} & & 1 & 2 & 0 & 0 & 0 & 0 & \multirow[b]{8}{*}{0.5937} & \multirow{8}{*}{27.75} & \multirow{8}{*}{26.83} & \multirow{8}{*}{0.92} & \multirow{8}{*}{0.35} \\
\hline & & 1 & 3 & 0 & 0 & 0 & 0 & & & & & \\
\hline & & 1 & 4 & 0 & 0 & 0 & & & & & & \\
\hline & $\mathrm{C}_{6}$ & 2 & 3 & 0 & 0 & 0 & 0.25 & & & & & \\
\hline & & 2 & 4 & 0 & 0.5 & 0 & 0.25 & & & & & \\
\hline & & 3 & 4 & -0.125 & 0.25 & 0.0156 & 0.0625 & & & & & \\
\hline & & 4 & - & -0.125 & - & 0.0156 & - & & & & & \\
\hline & & & & & $\sum$ & 0.0312 & 0.5625 & & & & & \\
\hline \multirow{8}{*}{7} & & 1 & 2 & -0.125 & 0 & 0.0156 & 0 & & & \multirow{8}{*}{25.58} & \multirow{8}{*}{-1.89} & \\
\hline & & 1 & 3 & -0.125 & 0.5 & 0.0156 & 0.25 & \multirow[b]{7}{*}{0.6249} & \multirow{7}{*}{23.69} & & & \\
\hline & & 1 & 4 & -0.125 & 0.25 & 0.0156 & 0.0625 & & & & & \\
\hline & $\mathrm{C}_{7}$ & 2 & 3 & 0 & 0 & 0 & 0 & & & & & -0.71 \\
\hline & & 2 & 4 & 0 & 0 & 0 & & & & & & \\
\hline & & 3 & 4 & & 0.5 & & 0.25 & & & & & \\
\hline & & 4 & - & -0.125 & - & 0.0156 & - & & & & & \\
\hline & & & & & $\sum$ & 0.0624 & 0.5625 & & & & & \\
\hline & & 1 & 2 & 0.375 & 0.75 & 0.1406 & 0.5625 & & & & & \\
\hline & & 1 & 3 & 0.375 & 0 & 0.1406 & & & & & & \\
\hline & & 1 & 4 & 0.375 & 0 & 0.1406 & 0 & & & & & \\
\hline 8 & $\mathrm{C}_{8}$ & 2 & 3 & -0.125 & 0 & 0.0156 & 0 & & 24.02 & 25.71 & -1.69 & 0.80 \\
\hline & & 2 & 4 & -0.125 & 0 & 0.0156 & 0 & & & & & \\
\hline & & 3 & 4 & & 0 & & 0 & & & & & \\
\hline & & 4 & - & 0 & 0 & 0 & 0 & & & & & \\
\hline & & & & & $\sum$ & 0.453 & 0.5625 & 1.0155 & & & & \\
\hline & & 1 & 2 & 0 & 0 & 0 & 0 & & & & & \\
\hline & & 1 & 3 & 0 & 0 & 0 & 0 & & & & & \\
\hline & & 1 & 4 & 0 & 0 & 0 & 0 & & & & & \\
\hline 9 & $\mathrm{C}_{9}$ & 2 & 3 & 0.375 & 0.75 & 0.1406 & 0.5625 & & 25.96 & 31.67 & -5.71 & -1.99 \\
\hline & & 2 & 4 & 0.375 & 0 & 0.1406 & & & & & & \\
\hline & & 3 & 4 & -0.125 & 0 & 0.0156 & 0 & & & & & \\
\hline & & 4 & - & 0 & - & 0 & - & & & & & \\
\hline & & & & & $\sum$ & 0.2968 & 0.5625 & 0.8593 & & & & \\
\hline & & 1 & 2 & 0 & 0 & 0 & 0 & & & & & \\
\hline & & 1 & 3 & 0 & 0 & 0 & 0 & & & & & \\
\hline & & 1 & 4 & 0 & 0 & 0 & 0 & & & & & \\
\hline 10 & $\mathrm{C}_{10}$ & 2 & 3 & -0.08 & 0.64 & 0.0064 & 0.4096 & & 26.57 & 27.77 & -1.2 & -0.45 \\
\hline & & 2 & 4 & -0.08 & 0.32 & 0.0064 & 0.1024 & & & & & \\
\hline & & 3 & 4 & -0.08 & 0.32 & 0.0064 & 0.1024 & & & & & \\
\hline & & 4 & - & -0.12 & - & 0.0144 & & & & & & \\
\hline 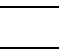 & & & & & $\sum$ & 0.0336 & 0.6144 & 0.648 & & & & \\
\hline
\end{tabular}

At significant level, $\alpha=0.05, \mathrm{t}_{\alpha / 1}(\mathrm{Ve})=\mathrm{t}_{0.05 / 10}=\mathrm{t}_{0.005(9)}=3.250$. The $\mathrm{t}-$ value is obtained from standard $\mathrm{t}-$ statistics table.

Since this is greater than any of the t- values calculated in (Table 3), we accept the Null hypothesis. Hence the model is adequate. 


\section{Fisher Test}

For this test, the parameter $\mathrm{y}$, is evaluated using the following equation:

$$
\mathrm{y}=\sum \mathrm{Y} / \mathrm{n}
$$

Where $\mathrm{Y}$ is the response and $\mathrm{n}$ the number of responses.

Using variance, $\mathrm{S}^{2}=[1 /(\mathrm{n}-1)]\left[\sum(\mathrm{Y}-\mathrm{y})^{2}\right]$ and $\mathrm{y}=\sum \mathrm{Y} / \mathrm{n}$ for $1 \leq \mathrm{i} \leq \mathrm{n}$

Table 4. F-Statistics for the controlled points

\begin{tabular}{|c|c|c|c|c|c|c|}
\hline $\begin{array}{l}\text { Response } \\
\text { Symbol }\end{array}$ & $\mathrm{Y}_{\text {(observed) }}$ & $\mathrm{Y}_{\text {(predicted) }}$ & $\mathrm{Y}_{(\mathrm{obs})}-\mathrm{y}_{(\mathrm{obs})}$ & $\mathrm{Y}_{(\mathrm{pre})}-\mathrm{y}_{(\mathrm{pre})}$ & $\left.\left(\mathrm{Y}_{(\mathrm{obs})}\right)^{-\mathrm{Y}}(\mathrm{obs})\right)^{2}$ & $\left(\mathrm{Y}_{(\mathrm{pre})^{-}} \mathrm{Y}_{(\mathrm{pre})}\right)^{2}$ \\
\hline $\mathrm{C}_{1}$ & 25.81 & 26.21 & -0.063 & -0.213 & 0.003969 & 0.045369 \\
\hline $\mathrm{C}_{2}$ & 26.39 & 25.85 & 0.517 & -0.573 & 0.267289 & 0.328329 \\
\hline $\mathrm{C}_{3}$ & 26.98 & 25.22 & 1.107 & -1.203 & 1.225449 & 1.447209 \\
\hline $\mathrm{C}_{4}$ & 23.32 & 23.38 & -2.553 & -3.043 & 6.517809 & 9.259849 \\
\hline $\mathrm{C}_{5}$ & 28.24 & 26.01 & 2.367 & -0.413 & 5.602689 & 0.170569 \\
\hline $\mathrm{C}_{6}$ & 27.75 & 26.83 & 1.877 & 0.407 & 3.523129 & 0.165649 \\
\hline $\mathrm{C}_{7}$ & 23.69 & 25.58 & -2.183 & -0.843 & 4.765489 & 0.710649 \\
\hline $\mathrm{C}_{8}$ & 24.02 & 25.71 & -1.853 & -0.713 & 3.433609 & 0.508369 \\
\hline $\mathrm{C}_{9}$ & 25.96 & 31.67 & 0.087 & 5.247 & 0.007569 & 27.53101 \\
\hline $\mathrm{C}_{10}$ & 26.57 & 27.77 & 0.697 & 1.347 & 0.485809 & 1.814409 \\
\hline Sum & 258.73 & 264.23 & & & 25.83281 & 41.98141 \\
\hline Mean & $\begin{array}{l}\mathrm{y}_{\text {(obs) }}= \\
25.873\end{array}$ & $\begin{array}{l}\mathrm{y}_{\text {(pre) }}= \\
26.423\end{array}$ & & & & \\
\hline
\end{tabular}

Therefore from (Table 4), $\mathrm{S}^{2}$ (obs) $=25.83281 / 9=2.87$ and $\mathrm{S}^{2}$ (pre) $=41.98141 / 9=4.66$

But the fisher test statistics is given by:

where $\mathrm{S}^{2}$ is the larger variance

$$
\mathrm{F}=\mathrm{S}_{1}^{2} / \mathrm{S}_{2}^{2}
$$

Hence $\mathrm{S}_{1}^{2}=4.66$ and $\mathrm{S}_{2}{ }_{2}=2.87$

Therefore, $\mathrm{F}=4.66 / 2.87=1.62$

From standard Fisher Table, $\mathrm{F}_{0.95}(9,9)=3.18$. Hence the regression equation is adequate.

Although the statistical tests were done for the same material (concrete), the grades are different because they were produced from different mixes obtained from the simplex analysis. However, if there were errors in the method used, the statistical tests will not agree to the acceptance of the model.

\section{Comparison of results}

The results obtained from the model were compared with those obtained from the experiment, as presented in Table 5

Table 5. Comparison of some Predicted Result with Experimental Results

\begin{tabular}{|l|l|l|l|}
\hline $\mathrm{S} / \mathrm{N}$ & $\begin{array}{l}\text { Experimental Result } \\
\left(\mathrm{N} / \mathrm{mm}^{2)}\right.\end{array}$ & $\begin{array}{l}\text { Predicted Result } \\
\left(\mathrm{N} / \mathrm{mm}^{2)}\right.\end{array}$ & Percentage Difference \\
\hline 1 & 25.81 & 26.21 & 1.55 \\
\hline 2 & 26.39 & 25.85 & 2.05 \\
\hline 3 & 26.98 & 25.22 & 6.52 \\
\hline 4 & 23.32 & 23.38 & 0.26 \\
\hline 5 & 27.75 & 26.83 & 3.32 \\
\hline
\end{tabular}

A comparison of the predicted results with the experimental results shows that the percentage difference ranges from a minimum of $0.26 \%$ to a maximum of $6.52 \%$, which is insignificant.

\section{Conclusion / recommendation}

(1) Scheffe's simplex method has been applied and used successfully to develop mathematical model for optimisation of concrete cube strength. However, the model applies to concrete of materials stated earlier. Interested researchers can learn the method and apply it to develop models for cube strength of concrete of different materials and sources.

(2) Concrete cube strength is a function of the proportions of the ingredients (cement, water, sand and coarse aggregate) of the concrete. 
(3) The student's t-test and the fisher test used in the statistical hypothesis showed that the model developed is adequate. Although the statistical tests were done for the same material (concrete), the grades are different because they were produced from different mixes obtained from the simplex analysis. However, if there were errors in the method used, the statistical tests will not agree to the acceptance of the model.

(4) The maximum concrete cube strength with the model is $31.71 \mathrm{Nmm}^{-2}$

(5) Since the maximum percentage difference between the experimental result and the predicted result is insignificant (i.e. 6.52), the optimisation model will yield accurate values of concrete cube strength if given the mix proportions and vice versa.

\section{References}

[1] Neville, A.M., Properties of Concrete, third edition, Pitman, England, 1981.

[2] Teychenne, D.C., Franklin, R.E.and Erntroy, H.C., Design of Normal Concrete Mixes, A Publication of Building Research Establishment, Transport and Road Research Laboratory and Cement and Concrete Association, 1975.

[3] Majid, K.I., Optimum Design of Structures, Butterworth \& Co. Ltd., London, 1974.

[4] British Standards Institution, BS 12: 1978, Specification for Portland Cement, 1978.

[5] British Standards Institution, BS 882: 1992, Specification for aggregates from natural sources for concrete, 1992.

[6] Scheffe, H., Experiments with Mixtures, Royal StatisticalSociety Journal, 20 (B), 1958, pp 344-360. 